Check for updates

Cite this: Chem. Sci., 2017, 8, 3885

Received 1st February 2017

Accepted 15th March 2017

DOI: $10.1039 / \mathrm{c} 7 \mathrm{sc} 00483 \mathrm{~d}$

rsc.li/chemical-science

\title{
Divergent reactivities in fluoronation of allylic alcohols: synthesis of Z-fluoroalkenes via carbon- carbon bond cleavage $\uparrow$
}

\author{
Tang-Lin Liu, Ji'En Wu and Yu Zhao* \\ An unconventional cleavage of an unstrained carbon-carbon bond in allylic alcohols can be induced by the \\ use of $\mathrm{N}$-fluorobenzenesulfonimide (NFSI) under catalyst-free conditions. By using this simple procedure, \\ a wide range of functionalized $Z$-fluoroalkenes can be accessed in high yield and selectivity from cyclic and \\ acyclic allylic alcohols.
}

\section{Introduction}

The incorporation of fluorine in pharmaceuticals and agrochemicals has become common practice due to the attractive properties that fluorine can provide. ${ }^{1}$ Accordingly, fluorination chemistry has been extensively explored in the last few decades to deliver fluorinated compounds with various substitution patterns. ${ }^{2}$ Despite the great progress achieved for catalytic $\mathrm{C}_{\mathrm{sp}^{3-}}$ F bond formation ${ }^{3}$ as well as aryl fluoride synthesis, ${ }^{4}$ significant limitations still exist in the preparation of certain structures such as fluoroalkenes that are highly valuable as peptide isosteres and building blocks. ${ }^{5}$ The preparation of fluoroalkenes through direct $\mathrm{C}-\mathrm{F}$ formation requires the use of sensitive organometallic reagents or intermediates (such as alkenyl lithium) and is thus limited in substrate scope. ${ }^{6}$ Alternatively, classical olefination reactions using fluorine-containing reagents have been extensively explored $;^{7}$ the control of olefin geometry in these methods, however, has remained an unsolved challenge. Only very recently the synthesis of 1,2-disubstituted $Z$-fluoroalkenes was achieved through catalytic cross metathesis. ${ }^{8}$ We present here a highly efficient and operationally simple method that can deliver functionalized trisubstituted $Z$ fluoroalkenes from readily available allylic alcohols with excellent stereoselectivity.

Electrophilic fluorination of enolate intermediates and alkenes has proven to be a highly successful strategy for C-F bond formation. ${ }^{3}$ By utilizing allylic alcohols as the substrate, a tandem metal-catalyzed isomerization to the enolate followed by fluorination has been reported to prepare $\alpha$-fluoroketones. ${ }^{9}$ Alternatively, fluorination of an alkene moiety followed by semipinacol rearrangement was realized to prepare $\beta$-fluoroketones

Department of Chemistry, National University of Singapore, 3 Science Drive 3, 117543, Republic of Singapore. E-mail: zhaoyu@nus.edu.sg

$\dagger$ Electronic supplementary information (ESI) available: Full data for the optimization of reaction conditions, detailed experimental procedures, and full characterization of substrates and products. See DOI: 10.1039/c7sc00483d
(Scheme 1a). ${ }^{10}$ Related to this, the fluorination induced ring opening of cyclopropanols was also reported recently to deliver $\beta$-fluoroketones using $\mathrm{Ag} / \mathrm{Cu}$ or photo-catalysis (Scheme $1 \mathrm{~b}$ ). ${ }^{11}$ We have discovered a general and catalyst-free fluorination of allylic alcohols simply by reacting the substrate with commercial NFSI open to air (Scheme 1c). In particular, a conceptually new transformation of allylic alcohols to $Z$-fluoroalkenes was realized through the cleavage of a non-activated carbon-carbon bond. It is noteworthy that carbon-carbon bond activation has been actively investigated as a new strategy in organic synthesis, with the majority of systems involving a transition metalmediated opening of strained C-C bonds. ${ }^{12}$ While examples of the activation of unstrained $\mathrm{C}-\mathrm{C}$ bonds in carbonyl derivatives

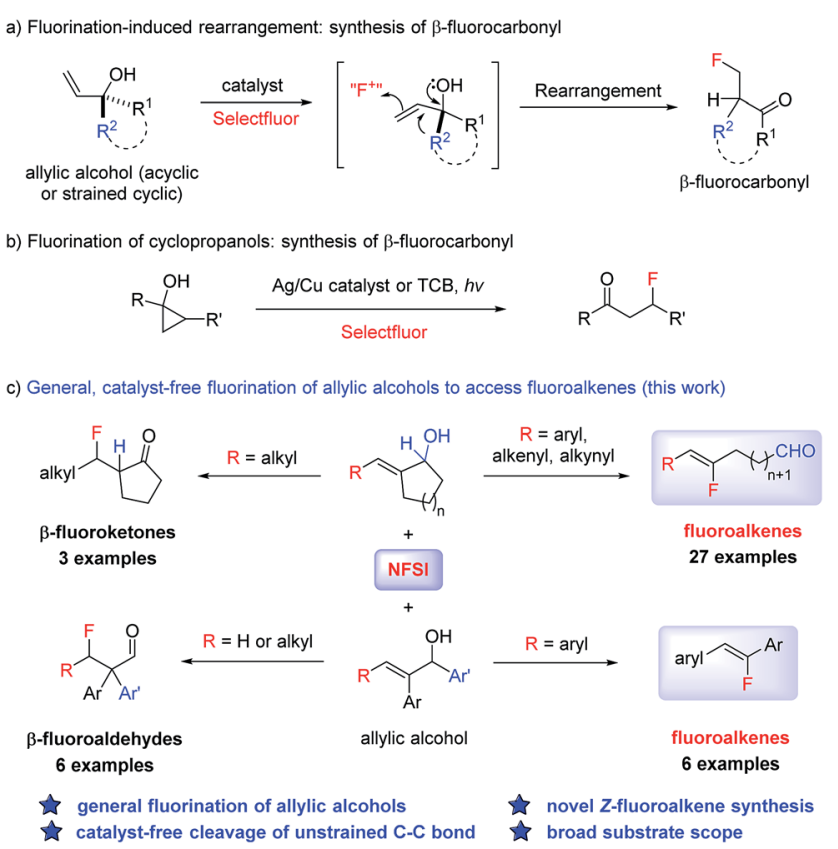

Scheme 1 Fluorination of allylic alcohols and cyclopropanols. 
are known under transition metal- or photocatalytic conditions,$^{13}$ our method represents an unprecedented reactivity of allylic alcohols, in which the non-activated $\mathrm{C}-\mathrm{C}$ bond undergoes cleavage induced by NFSI. The stereoselective formation of fluoroalkenes also renders this system a valuable tool in chemical synthesis.

\section{Results and discussion}

In the past few years, our laboratory has been exploring catalytic enantioselective redox-neutral processes for the preparation of important chiral entities. ${ }^{\mathbf{1 4}}$ During our investigation of a Rhcatalyzed isomerization of allylic alcohol 1a to the corresponding $\alpha$-benzyl cyclopentanone (Scheme 2), ${ }^{14 e}$ as a mechanistic probe we attempted the synthesis of $\mathbf{3 a}$ by trapping the proposed enolate intermediate with an electrophilic fluorinating reagent such as NFSI. To our great surprise, no 3a could be accessed under this set of conditions and a completely unexpected product 2a was formed instead. This transformation involves the cleavage of an unstrained carboncarbon (C-C) bond in a five-membered ring and delivers synthetically versatile fluoroalkene $\mathbf{2 a}$ as a single $Z$-isomer (determined by the $J_{\mathrm{H}-\mathrm{F}}$ value as well as $2 \mathrm{D} \mathrm{NMR}$; see the ESI $\dagger$ for details). Intrigued by this discovery, we decided to explore this transformation in detail.

As summarized in Table 1, a series of transition metal complexes based on $\mathrm{Rh}, \mathrm{Ir}, \mathrm{Ru}$, etc. were examined first. Interestingly, all these catalysts led to the formation of $\mathbf{2 a}$ in similar yields of $58-60 \%$ (entries $1-3$ ). The addition of ligands such as triphenylphosphine had no effect at all (results not shown). When the reaction was carried out by simply mixing 1a and NFSI at ambient temperature in the absence of any catalyst, a $61 \%$ yield was obtained for 2 a (entry 4 ). Clearly this intriguing $\mathrm{C}-\mathrm{C}$ bond cleavage of allylic alcohols proceeds under transition metal-free conditions. The use of other fluorinating reagents such as Selectfluor provided no conversion to $2 \mathbf{a}$ at all (entry 5). The effect of various bases as the additive was examined next, which led to reduced reaction efficiency (e.g. use of $\mathrm{K}_{2} \mathrm{CO}_{3}$ in entry 6). Considering the important precedent on photocatalyzed fluorination reactions from the Lectka group, ${ }^{13 b}$ we tested the effect of light in our reaction by carrying out the same reaction in the dark. The same reaction efficiency was obtained, thus excluding the possibility of photocatalysis (entry 7). When $\mathrm{Ag}$ - or $\mathrm{Cu}$-based salts that are known to promote single electron transfer were examined, no improvement was observed for our reaction either (entries 8-9). ${ }^{\mathbf{1 1}}$

To further optimize the reaction, the loading of NFSI and the reaction temperature were varied. The use of 2.0 equiv. of NFSI

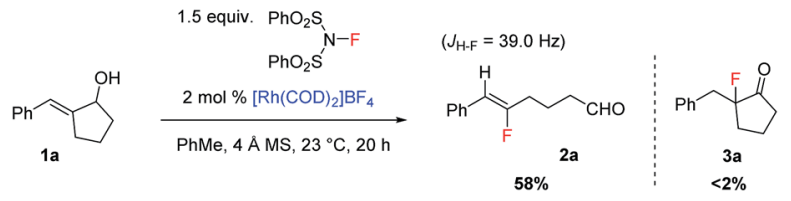

Scheme 2 Discovery of fluoroalkene synthesis from allylic alcohols.
Table 1 Optimization of fluoroalkene synthesis ${ }^{a}$

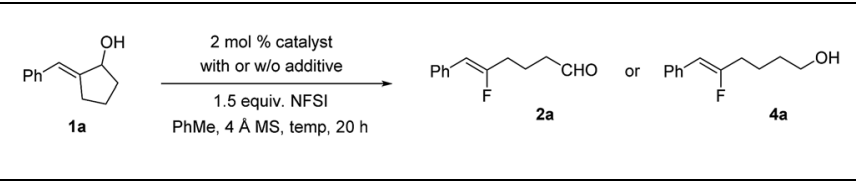

\begin{tabular}{|c|c|c|c|c|}
\hline Entry & Catalyst & $\mathrm{F}^{+}$(equiv.) & $\begin{array}{l}T \\
\left({ }^{\circ} \mathrm{C}\right)\end{array}$ & Yield $^{b}(\%)$ \\
\hline 1 & {$\left[\mathrm{Rh}(\operatorname{cod})_{2}\right] \mathrm{BF}_{4}$} & NFSI (1.5) & 23 & 58 \\
\hline 2 & {$[\operatorname{Ir}(\operatorname{cod}) \mathrm{Cl}]_{2}$} & NFSI (1.5) & 23 & 60 \\
\hline 3 & {$\left[\operatorname{RuCl}_{2}(p \text {-cymeme })\right]_{2}$} & NFSI (1.5) & 23 & 59 \\
\hline 4 & - & NFSI (1.5) & 23 & 61 \\
\hline 5 & - & Selectfluor & 23 & $<5$ \\
\hline 6 & $\mathrm{~K}_{2} \mathrm{CO}_{3}$ & NFSI (1.5) & 23 & 55 \\
\hline 7 & In the dark & NFSI (1.5) & 23 & 60 \\
\hline 8 & $\mathrm{Ag}_{2} \mathrm{CO}_{3}$ & NFSI (1.5) & 23 & 54 \\
\hline 9 & $\mathrm{CuCl}$ & NFSI (1.5) & 23 & 50 \\
\hline 10 & - & NFSI (1.5) & 40 & 66 \\
\hline 11 & - & NFSI (2.0) & 40 & 70 \\
\hline 12 & - & NFSI (2.0) & 40 & $82(4 \mathbf{a})^{c}$ \\
\hline
\end{tabular}

${ }^{a}$ The reactions were carried out with $0.2 \mathrm{mmol}$ of $1 \mathrm{a}$ and $100 \mathrm{mg}$ of a $4 \AA$ molecular sieve in $2 \mathrm{~mL}$ of toluene open to air using commercially available NFSI. ${ }^{b}$ Isolated yields. ${ }^{c}$ The isolated yield of alcohol 4 a after the reduction of the aldehyde.

at $40{ }^{\circ} \mathrm{C}$ proved to be optimal (entries 10-11). Overall, a simple and effective procedure that involves simple mixing of the substrate and NFSI with gentle heating proved to be the most effective. Finally, a more convenient procedure was adopted that included an in situ reduction of 2 a using $\mathrm{NaBH}_{4}$; the corresponding primary alcohol $\mathbf{4 a}$ could be isolated in a high yield of $82 \%$ (entry 12). The scale-up of this reaction proved to be straightforward as well. A similar yield of $78 \%$ was obtained for 4a on a $5 \mathrm{mmol}$ scale (Scheme 3).

With the optimal conditions in hand, we moved on to explore the scope of this ring-opening fluorination reaction. For the ring-opening of cyclopentanols $(n=1)$, various substituted aryl groups can be well-tolerated (Scheme 3a). A wide range of fluoroalkenes bearing electron-deficient (4b-4e), electronneutral (4f-g) and electron-rich (4h) substitutions at the paraposition of the arene were prepared in good to excellent yield. Reactive functionalities such as ester or cyano groups could be well tolerated to produce $\mathbf{4 i - 4} \mathbf{j}$ in good yield as single isomers. meta-Substituted arenes such as $\mathbf{4 k}$ were produced similarly well. For ortho-substituted substrates (41-4n), only moderate yields were obtained probably due to the steric hindrance of these substrates. Naphthyl- and heterocycle-substituted fluoroalkenes (4o-4r) could also be prepared in reasonable yields. While alkyl-substituted allylic alcohols failed to produce the desired products, alkenyl- and alkynyl-substituted substrates underwent fluorination smoothly to produce fluoro-substituted diene $4 \mathbf{s}$ and enyne $\mathbf{4 t}$. Such highly functionalized fluoroalkenes are accessed for the first time and could prove highly valuable as building blocks in chemical synthesis.

In addition to cyclopentanols, this fluorination method is also applicable to the opening of different sized rings, including 4, 6, 7, and 8-membered substrates (Scheme 3b). In this way 


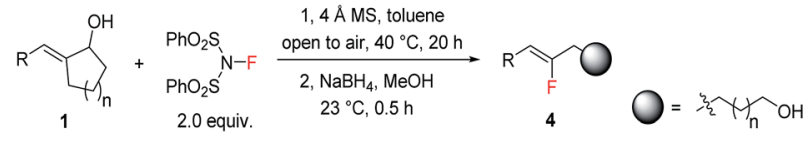

a) $n=1$

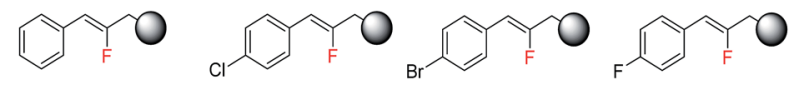

$4 a$
$82 \%, Z / E=>20: 1$

$(78 \%, Z / E=>20: 1)^{a}$
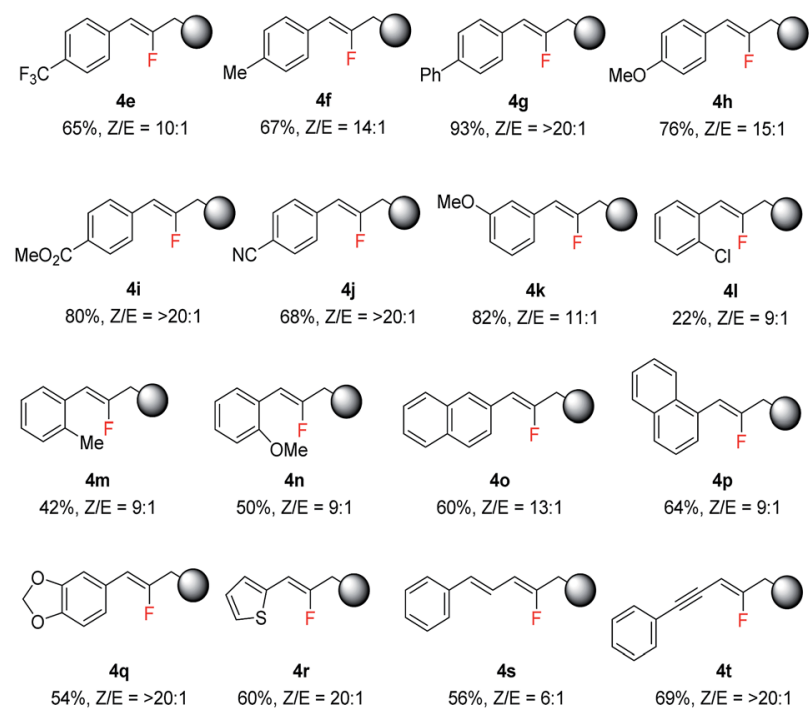

b) $n=0-4$

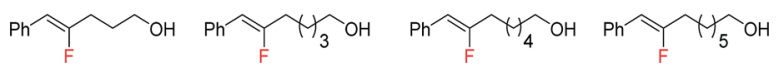

$4 u(n=0) \quad 4 v(n=2) \quad 4 w(n=3) \quad 4 x(n=4)$

$83 \%$, ZIE $=5: 1 \quad 56 \%$, ZIE $=>20: 1 \quad 45 \%$, ZIE $=>20: 1 \quad 42 \%$, ZIE $=>20: 1$

c)
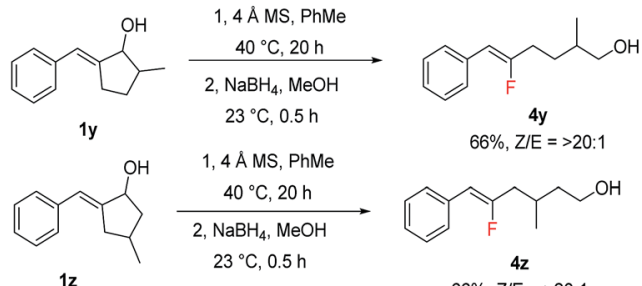

$66 \%$, Z/E $=>20: 1$

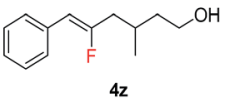

$66 \%, Z / E=>20: 1$

d) bis-ring opening fluorination

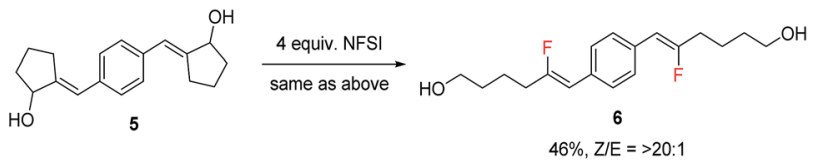

Scheme 3 Scope of fluorination-induced ring opening of cyclic allylic alcohols. See the ESI† for the detailed procedure. The product Z/E ratios were determined by crude NMR. All yields are isolated yields. a $5 \mathrm{mmol}$ scale reaction.

a series of fluoroalkenes bearing a formyl or alcohol functionality at different distances can be accessed conveniently, although the efficiency gradually drops for longer tethers $(\mathbf{4 u}-$ $\mathbf{4 x}$ ). In addition to the generation of simple linear products, substitution at different positions in the cycloalkanol substrates (such as $\mathbf{1 y}$ and $\mathbf{1 z}$ ) was well-tolerated to produce $\alpha$ - or $\beta$ branched fluoroalkene-containing alcohols $4 y$ and $4 \mathbf{z}$ with excellent $Z$-selectivity (Scheme 3c).

Finally, double fluorination of bis-allylic alcohol 5 also proceeded smoothly to produce bis-fluoroalkene $\mathbf{6}$ in moderate yield as a single isomer (Scheme 3d). This transformation, however, failed to produce ketone products by the reaction of tertiary allylic alcohols related to 1a. Although tertiary alcohols are more popular substrates in carbon-carbon bond activation, in our case the greater steric hindrance in these substrates is likely the culprit for this lack of reactivity.

The success of opening large-ring substrates suggested that acyclic substrates might undergo a similar transformation as well. Substituted acyclic allylic alcohols were examined next. As shown in Scheme 4a, this type of substrate underwent the same $\mathrm{C}-\mathrm{C}$ bond cleavage to produce trisubstituted fluoroalkenes, ${ }^{7,8}$ while producing benzaldehyde as the side product. It is noteworthy that the alternative semi-pinacol rearrangement product (Scheme 1b) was not observed at all. The efficiency of this process, however, leaves much room for further optimization. While 8a bearing an electron-rich aryl substituent was obtained in a good yield of $70 \%$, most fluoroalkenes were produced in yields ranging from $14-33 \%$. The stereoselectivity of this process, however, is uniformly high to produce the fluoroalkenes as a single $Z$-isomer. Related fluoroalkenes of the same substitution pattern have been popular targets in medicinal chemistry ${ }^{15}$ and our method represents an alternative method for their synthesis with unparalleled stereoselectivity.

In addition, this fluorination of allylic alcohols can be extended to benzylic alcohols (Scheme $4 \mathrm{~b}$ ). A proof-of-principle reaction involves the fluorination of indole-substituted benzylic alcohol 9, which produced 3 -fluoroindole 10 in $24 \%$ yield. This greatly expanded the scope of this fluorination method and further application is currently under investigation.

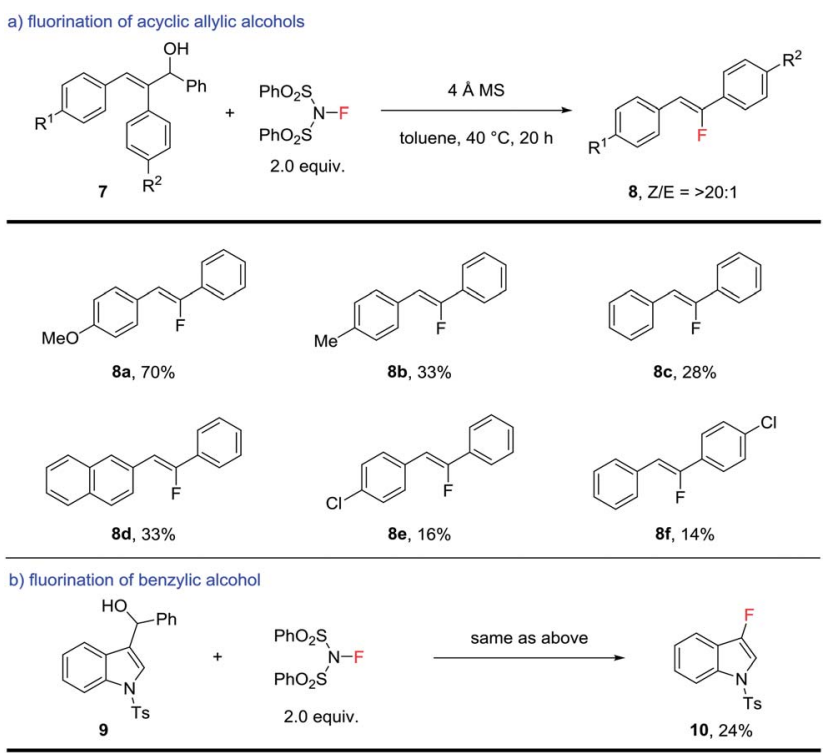

Scheme 4 Scope of fluorination of acyclic allylic and benzylic alcohols. 
It is worth noting that an aryl (or $\mathrm{sp}^{2}$-hybridized) substituent on the alkene moiety of the allylic alcohol substrates is required for the synthesis of fluoroalkenes as shown in Schemes 3 and 4 . When allylic alcohols bearing an alkyl substituent were examined (11 or 12), an intriguing and complete change in reactivity was observed (Scheme 5). In the case of cyclopentanols 11, fluorination under identical conditions led to the formation of $\beta$-fluoroketones 13a-c in moderate to good yields, which should be formed via $\beta$-fluorination followed by a hydride migration. In contrast, the acyclic allylic alcohols 12 underwent $\beta$-fluorination followed by a more classical semi-pinacol rearrangement to deliver $\beta$-fluoroaldehydes 14a-f in uniformly good yields. ${ }^{10}$ While these $\beta$-fluoroketones $\mathbf{1 3}$ and $\mathbf{1 4}$ are valuable compounds on their own, their synthesis also provided important insight into the mechanism of this catalyst-free fluorination of allylic alcohols. More intriguingly, a small amount of fluoroalkene 8a was obtained during the synthesis of 14e and 14f. The formation of $\mathbf{8 a}$ from 12, a formal cleavage of the alkene moiety of the substrate, led us to hypothesize the involvement of a key oxetane intermediate in the fluoroalkene synthesis.

In an effort to better understand the limitations in the fluorination of acyclic allylic alcohols, the reaction of $7 \mathrm{~g}$ was carried out on a larger scale and the product mixture was analyzed carefully (Scheme 6a). In addition to the desired fluoroalkene $\mathbf{8 g}$, $\beta$-fluoroaldehyde $\mathbf{1 7}$ was isolated in a low yield, indicating that two fluorination pathways were in competition. More interestingly, a small amount of oxetane $\mathbf{1 5}$ and the alternative $Z$-fluoroalkene $\mathbf{1 6}$ was also isolated, which could be linked to the formation of $\mathbf{8 a}$ from the fluorination of $\mathbf{1 2 e} / \mathbf{1 2 f}$ as shown in Scheme 5.

When oxetane 15 was subjected to the fluorination conditions (Scheme 6b), however, only a slow conversion to 16 was

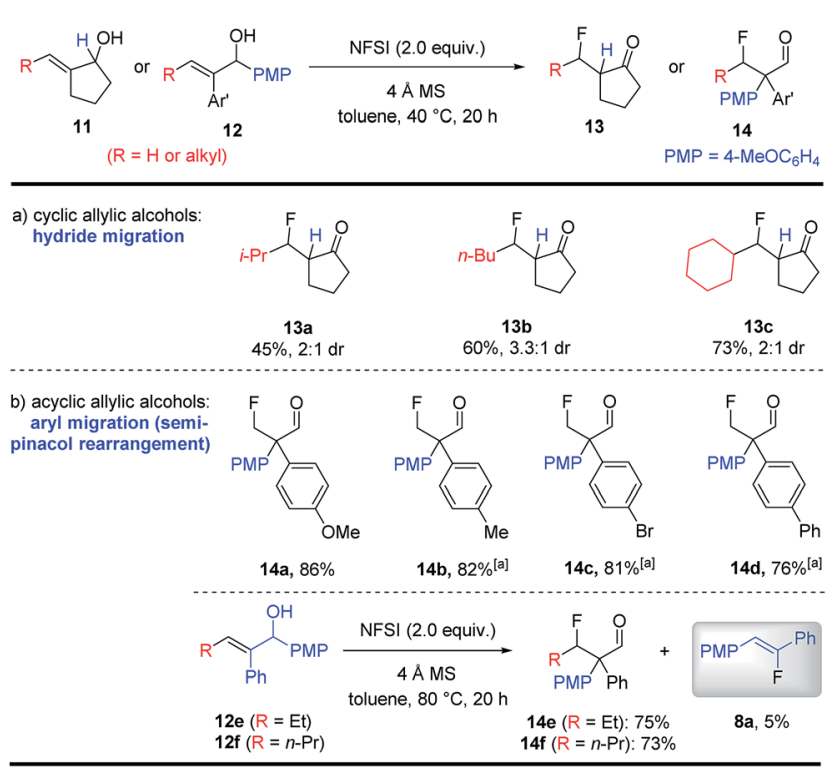

Scheme 5 Fluorination of alkyl-substituted allylic alcohols. See the $\mathrm{ESI} \dagger$ for the detailed procedure. The product d.r.s were determined by crude NMR. All yields are isolated yields. ${ }^{a}$ The reaction was carried out at $80^{\circ} \mathrm{C}$

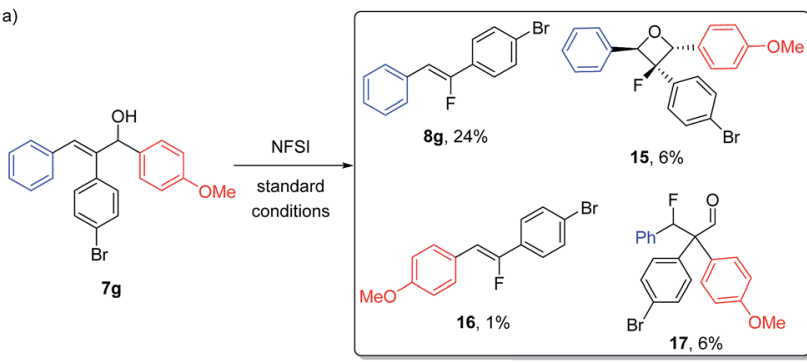

b)
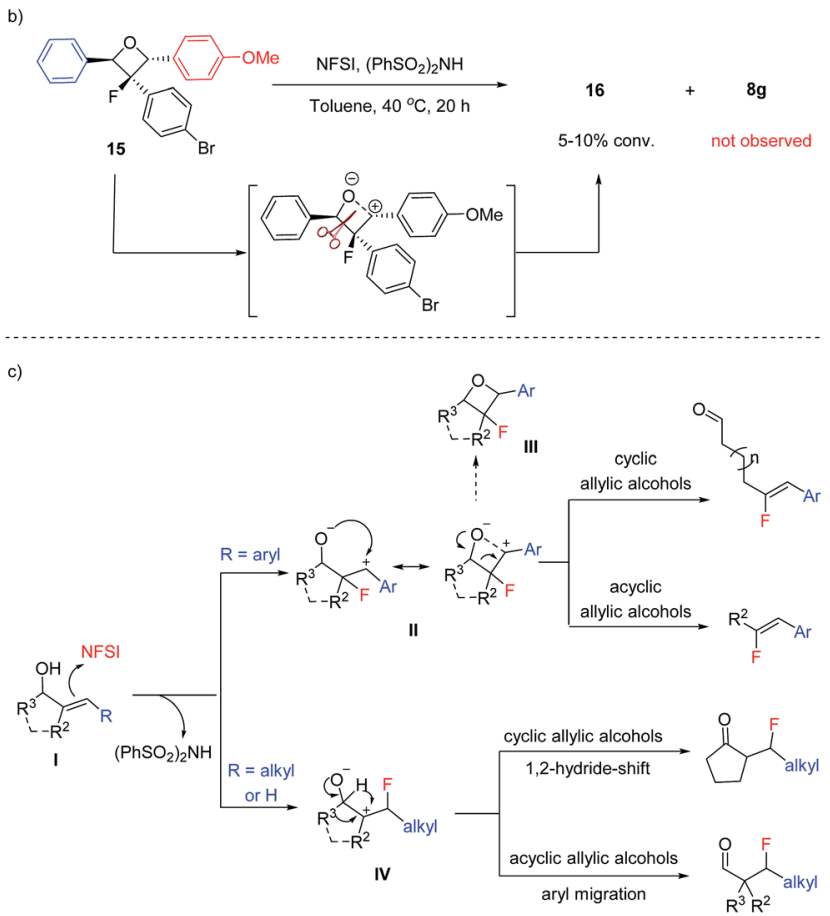

Scheme 6 Preliminary mechanistic studies and the proposed reaction pathway.

observed; no formation of $\mathbf{8 g}$ could be detected. We hypothesized that the conversion of $\mathbf{1 5}$ to $\mathbf{1 6}$ likely proceeded through a zwitterionic intermediate and a carbocation is formed preferentially on the more electron-rich benzylic position, which explained why $\mathbf{8 g}$ was not formed. This observation also indicated that oxetane formation is likely a side reaction pathway in the fluoroalkene synthesis instead of being an intermediate for the formation of $\mathbf{8 g}$ or other fluoroalkenes shown in Scheme 4 .

Based on the above observations and taking all of the types of fluorination products into consideration, we propose the general mechanism for fluorination of allylic alcohols using NFSI as shown in Scheme 6c. For the substrates bearing an aryl substituent (or alkenyl and alkynyl as in $\mathbf{5 s}$ and $\mathbf{5 t}$ ), the electrophilic fluorination generates a zwitterion II, in which the formation of a more stable benzylic carbocation serves as the driving force for this regio-selectivity. Fragmentation of II then leads to the formation of fluoroalkenes. Oxetane formation (III) is a side pathway of this reaction, and oxetane could undergo fragmentation through zwitterion II or the alternative species to deliver the more electron-rich fluoroalkene product $(e . g ., 16) .{ }^{16}$ For the substrates bearing an alkyl substituent, on the other 


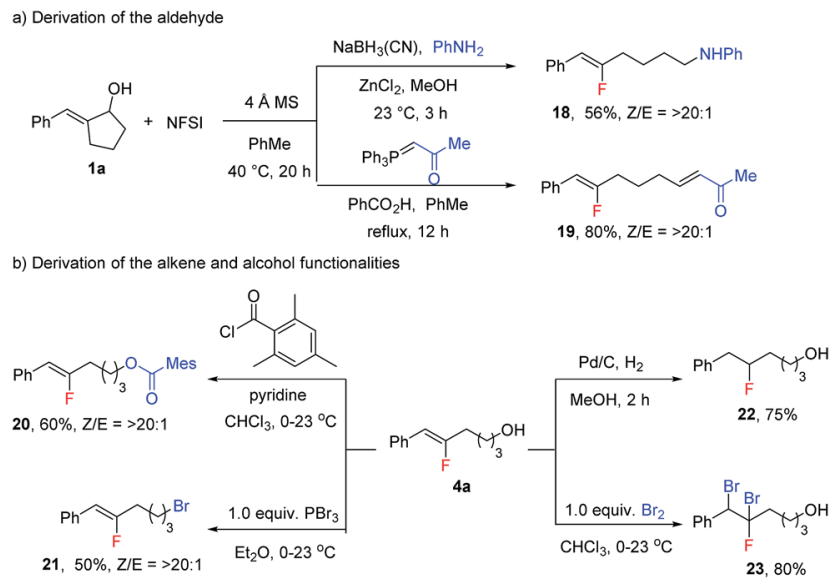

Scheme 7 Derivatization of fluoroalkenes.

hand, the electrophilic fluorination takes place at the $\beta$-position to generate zwitterion IV. Again, the stability of the carbocation is likely the determining factor. A migration (of either a hydride or aryl group) will then follow to deliver the more traditional $\beta$ fluorocarbonyl products.

The products from this reaction can be used as versatile building blocks to prepare various organofluorine compounds (Scheme 7). In addition to the reduction of 2a to yield alcohol 4a, reductive amination or Wittig olefination of 2a proceeded smoothly in one pot with fluorination to yield amine 18 or enone 19 in good yields (Scheme 7a). The alcohol functionality in $4 \mathbf{a}$ could also be converted to ester or bromide functionalities as in $\mathbf{2 0}$ and $\mathbf{2 1}$ without optimization (Scheme $7 \mathrm{~b}$ ). More importantly, the fluoroalkene moiety has proven to be a synthetically versatile building block to access a diverse range of fluoro-containing compounds. ${ }^{17}$ As representative examples, hydrogenation and dibromination of $\mathbf{4 a}$ were carried out to produce alkyl fluoride $\mathbf{2 2}$ and multiple-halogen-containing $\mathbf{2 3}$.

\section{Conclusions}

We have discovered a general fluorination of allylic alcohols and in particular, a conceptually new and practical method to access functionalized $Z$-fluoroalkenes with good to excellent geometry control. This operationally simple procedure involves the reaction of readily available allylic alcohols and NFSI open to air with gentle heating to produce the versatile functionalized fluoroalkenes. Current efforts in our laboratory are focused on the application of this method to the preparation of other valuable fluorinated compounds.

\section{Acknowledgements}

We are grateful for the generous financial support from the Singapore National Research Foundation (NRF Fellowship R143-000-477-281) and the Ministry of Education (MOE) of Singapore (R-143-000-613-112).

\section{Notes and references}

1 For selected recent reviews, see: $(a)$ K. Müller, C. Faeh and F. Diederich, Science, 2007, 317, 1881; (b) S. Purser, P. R. Moore, S. Swallow and V. Gouverneur, Chem. Soc. Rev., 2008, 37, 320; (c) M. Cametti, B. Crousse, P. Metrangolo, R. Milani and G. Resnati, Chem. Soc. Rev., 2012, 41, 31.

2 (a) T. Furuya, A. S. Kamlet and T. Ritter, Nature, 2011, 473, 470; (b) T. Liang, C. N. Neumann and T. Ritter, Angew. Chem., Int. Ed., 2013, 52, 8214; (c) A. F. Brooks, J. J. Topczewski, N. Ichiishi, M. S. Sanford and P. J. H. Scott, Chem. Sci., 2014, 5, 4545.

3 For a selected recent review, see: X. Yang, T. Wu, R. J. Phipps and F. D. Toste, Chem. Rev., 2015, 115, 826.

4 For a selected recent review, see: M. G. Campbell and T. Ritter, Chem. Rev., 2015, 115, 612.

5 For a selected review on fluoroalkenes, see: $(a)$ S. CouveBonnaire, D. Cahard and X. Pannecoucke, Org. Biomol. Chem., 2007, 5, 1151. For selected reviews on fluoroalkene synthesis, see: $(b)$ D. J. Burton, Z.-Y. Yang and W. Qiu, Chem. Rev., 1996, 96, 1641; (c) J. H. van Steenis and A. V. der Gen, J. Chem. Soc., Perkin Trans. 1, 2002, 2117; (d) G. Landelle, M. Bergeron, M.-O. Turcotte-Savard and J.-F. Paquin, Chem. Soc. Rev., 2011, 40, 2867.

6 M.-H. Yang, S. S. Matikonda and R. A. Altman, Org. Lett., 2013, 15, 3894, and references therein.

7 For selected reviews on fluoroalkene synthesis via olefination, see: (a) B. Zajc and R. Kumar, Synthesis, 2010, 1822; (b) E. Pfund, T. Lequeux and D. Gueyrard, Synthesis, 2015, 47, 1534; (c) Y. Zhao, F. Jiang and J. Hu, J. Am. Chem. Soc., 2015, 137, 5199; (d) W. Zhang, W. Huang and J. Hu, Angew. Chem., Int. Ed., 2009, 48, 9858.

8 M. J. Koh, T. T. Nguyen, H. Zhang, R. R. Schrock and A. H. Hoveyda, Nature, 2016, 531, 459.

9 N. Ahlsten and B. Martin-Matute, Chem. Commun., 2011, 47, 8331.

10 (a) B. Wang and Y. Q. Tu, Acc. Chem. Res., 2011, 44, 1207; (b) F. Romanov-Michailidis, L. Guénée and A. Alexakis, Angew. Chem., Int. Ed., 2013, 52, 9266.

11 (a) H. Zhao, X. Fan, J. Yu and C. Zhu, J. Am. Chem. Soc., 2015, 137, 3490; (b) S. Bloom, D. D. Bume, C. R. Pitts and T. Lectka, Chem.-Eur. J., 2015, 21, 8060; (c) S. Ren, C. Feng and T.-P. Loh, Org. Biomol. Chem., 2015, 13, 5105.

12 For selected reviews, see: (a) G. Dong, Topics in Current Chemistry, Springer, 2014; (b) I. Marek, A. Masarwa, P.-O. Delaye and M. Leibeling, Angew. Chem., Int. Ed., 2015, 54, 414; (c) L. Souillart and N. Cramer, Chem. Rev., 2015, 115, 9410.

13 For a selected review, see: $(a)$ F. Chen, T. Wang and N. Jiao, Chem. Rev., 2014, 114, 8613.For a recent elegant example of fluorination-induced photo-catalytic cleavage of unstrained C-C bonds, see: (b) C. R. Pitts, M. S. Bloom, D. D. Bume, Q. A. Zhang and T. Lectka, Chem. Sci., 2015, 6, 5225.

14 (a) Y. Zhang, C.-S. Lim, D. S. B. Sim, H.-J. Pan and Y. Zhao, Angew. Chem., Int. Ed., 2014, 53, 1399; (b) Z.-Q. Rong, 
Y. Zhang, R. H. B. Chua, H.-J. Pan and Y. Zhao, J. Am. Chem. Soc., 2015, 137, 4944; (c) H.-J. Pan, T. W. Ng and Y. Zhao, Chem. Commun., 2015, 51, 11907; (d) L.-C. Yang, Y.-N. Wang, Y. Zhang and Y. Zhao, ACS Catal., 2017, 7, 93; (e) T.-L. Liu, T. W. Ng and Y. Zhao, J. Am. Chem. Soc., 2017, 139, 3643.

15 D. Alloatti, G. Giannini, W. Cabri, I. Lustrati, M. Marzi, A. Ciacci, G. Gallo, M. O. Tinti, M. Marcellini, T. Riccioni, M. B. Guglielmi, P. Carminati and C. Pisano, J. Med. Chem., 2008, 51, 2708.
16 (a) J. R. Ludwig, P. M. Zimmerman, J. B. Gianino and C. S. Schindler, Nature, 2016, 533, 374; (b) L. Ma, W. Li, H. Xi, X. Bai, E. Ma, X. Yan and Z. Li, Angew. Chem., Int. Ed., 2016, 55, 10410.

17 For selected examples, see: (a) M. Engman, J. S. Diesen, A. Paptchikhine and P. G. Andersson, J. Am. Chem. Soc., 2007, 129, 4536; (b) O. A. Wong and Y. Shi, J. Org. Chem., 2009, 74, 8377. 